\title{
Digitale Potenziale nutzen und gestalten
}

Die Digitalisierung ist Treiber eines Strukturwandels, der Wirtschaft und Gesellschaft dynamisch verändert. Die Chancen und Risiken dieser Entwicklung werden vielfach diskutiert, und es stellt sich die Frage, in welchen Bereichen Veränderungen stattfinden, die als Potenziale identifiziert werden können, aber auch, inwiefern potenzielle Risiken dieser Entwicklungen mit geeigneten Maßnahmen abgemildert oder kompensiert werden können. Dabei können wirtschaftspolitische Weichenstellungen vorgenommen werden, um digitale Potenziale zu nutzen, aber auch, um Verlierer dieser Entwicklung zu kompensieren und damit mögliche soziale Verwerfungen zu entschärfen. Auf der gemeinsamen Konferenz von Wirtschaftsdienst/ZBW und dem Institut für Arbeitsmarkt- und Berufsforschung am 2. Dezember 2019 in Berlin wurde analysiert und diskutiert, wie sich digitale Potenziale nutzen und gestalten lassen und welche Herausforderungen der Wirtschaftspolitik angesichts des umfassenden digitalen Wandels gegenüberstehen. Auf dieser Grundlage ist das nun vorliegende Heft mit den Beiträgen zur Konferenz entstanden.

Den Auftakt machen Thomas Franke und Mourad Zoubir von der Universität zu Lübeck mit ihrem Beitrag „Technology for the People? Humanity as a Compass for the Digital Transformation". Sie stellen der Möglichkeit eines innovationsgetriebenen digitalen Wandels einen humanzentrierten Ansatz gegenüber, d.h. technologische Innovationen sollen aus der Gesellschaft heraus gesteu-

(c) Der/die Autor(en) 2020. Open Access: Dieser Artikel wird unter der Creative Commons Namensnennung 4.0 International Lizenz (http:// creativecommons.org/licenses/by/4.0/deed.de) veröffentlicht.

Open Access wird durch die ZBW - Leibniz-Informationszentrum Wirtschaft gefördert.

Kristin Biesenbender, M.A., ist Redakteurin beim Wirtschaftsdienst in der ZBW - LeibnizInformationszentrum Wirtschaft in Hamburg.

Jun.-Prof. Dr. Christian Breuer ist Chefredakteur von Wirtschaftsdienst und Intereconomics in der ZBW.

Cora Wacker-Theodorakopoulos, Dipl.-Volkswirtin, ist Redakteurin beim Wirtschaftsdienst in der ZBW. ert und gestaltet werden. Denn Innovationen sollten den Menschen mit seinen physischen und psychischen Bedürfnissen ins Zentrum rücken und dabei auch eine ethische und gesellschaftlich wünschenswerte Perspektive einbeziehen. Ein humanzentrierter Ansatz stellt die Chancen der Digitalisierung in den Mittelpunkt, während Sorgen und Risiken eher in den Hintergrund treten.

Michael Hüther vom Institut der deutschen Wirtschaft befasst sich mit der Umsetzung der Digitalisierung auf Unternehmensebene. Diese ist längst praktischer Bestandteil strategischer Planungen und Handlungen. Mit Blick auf das Produktivitätswachstum ist zu erwarten, dass Steigerungen nur verzögert auftreten und in vielen Bereichen noch unausgeschöpfte Potenziale liegen. Positive Beschäftigungseffekte sind insbesondere im Business-toBusiness-Markt zu erwarten, da implizites, in Fachkräften gebundenes Fachwissen (noch längst) nicht durch Maschinen und Algorithmen zu ersetzen ist. Grundsätzlich wird es in zunehmendem Maße zu einer Veränderung der Kompetenzanforderungen kommen. Die Herausforderung besteht darin, weder weniger innovationsaffine Unternehmen noch weniger IT-affine Beschäftigte abzuhängen.

Justus Haucap vom Düsseldorf Institute for Competition Economics stellt in seinem Beitrag die Marktposition der Digitalunternehmen heraus, die besondere Wettbewerbsvorteile durch inr Agieren mit Plattformen, Netzwerken und Daten realisieren können. Dabei bestehen insbesondere auch Anreize und Möglichkeiten, in wettbewerbswidriger Weise den Markt zu verschließen. Die sich ausweitende Marktmacht großer Plattformökonomien macht eine Neujustierung des Kartellrechts erforderlich - insbesondere um den Schutz der Möglichkeiten zum Multi-Homing zu stärken. Die geplante 10. Novelle des Gesetzes gegen Wettbewerbsbeschränkungen legt inren Fokus dabei auf die Ausweitung der kartellrechtlichen Missbrauchsaufsicht und nicht auf die Fusionskontrolle.

Ina Schieferdecker und Christoph March vom Bundesministerium für Bildung und Forschung stellen den $\mathrm{Zu}-$ sammenhang von digitalen Innovationen und Technologiesouveränität in den Mittelpunkt ihres Beitrags. Technologiesouveränität ist eine zentrale Voraussetzung, um die digitale Transformation im Dialog mit der Gesellschaft und im Einklang mit ihren Werten zu gestalten. Dabei geht es um die Fähigkeit, Anforderungen an Technologien zu formulieren und diese am Markt durchzusetzen. Eine integrierte Forschungs- und Innovationspolitik muss 
die Entwicklung und den Transfer von Technologien und Zukunftskompetenzen gleichermaßen fördern und dabei insbesondere auch auf eine europäische Zusammenarbeit setzen.

Die Auswirkungen der Digitalisierung auf dem Arbeitsmarkt stehen im Fokus des Beitrags von Enzo Weber vom Institut für Arbeitsmarkt- und Berufsforschung. Er widmet sich dem Potenzial der „Digitalen Sozialen Sicherung“ für die Plattformarbeit. Gerade in der Plattformarbeit treten häufig prekäre Beschäftigungsverhältnisse auf, wobei auch insbesondere die soziale Absicherung gravierende Lücken aufweist. Die Herausforderung besteht darin, Elemente einer Sozialversicherung in einen sehr kurzfristig agierenden, auch globalen Markt zu integrieren. Das Konzept der „Digitalen Sozialen Sicherung“ sieht vor, in die Plattformen einen Mechanismus zu implementieren, der mit jeder Beendigung eines Jobs automatisch einen bestimmten Prozentsatz des vereinbarten Entgelts als Sozialbeitrag abführt. Damit wird ein effektives digitales Quellenabzugsverfahren für Plattformarbeit ermöglicht. Das System ist offen für die Beteiligung verschiedener Staaten, ohne die Souveränität und Flexibilität der nationalen Sozialversicherungen einzuschränken, da die Beiträge an die nationalen Sozialversicherungen weitergeleitet werden können.

Melanie Arntz, Terry Gregory und Ulrich Zierahn vom Leibniz-Zentrum für Europäische Wirtschaftsforschung analysieren die Digitalisierung und die Zukunft der Arbeit in ihrem Beitrag aus makroökonomischer Perspektive. Infolge der digitalen Transformation lassen sich bestimmte Tätigkeiten durch Maschinen und Algorithmen automatisieren. Diese zunehmenden Veränderungen schüren Befürchtungen, dass diese Entwicklung hohe Arbeitsplatzverluste nach sich zieht. Allerdings zeigen empirische Studien, dass die technologischen Potenziale zur Automatisierung von Jobs vielfach deutlich überschätzt werden. Die Möglichkeiten der Automatisierung werden längst nicht alle ausgeschöpft, denn in der betrieblichen Praxis können die Kosten den Nutzen der Automatisierung durchaus übersteigen. Auch muss sich die Arbeitsteilung zwischen Mensch und Maschine stets flexibel anpassen, woraus sich neue Tätigkeitsfelder ergeben. Der tiefgreifende Strukturwandel stellt die Arbeitskräfte vor neue Herausforderungen. Das macht es erforderlich, entsprechende Kompetenzen bei den Beschäftigten aufzubauen.

Saskia Montebovi und Alberto Barrio Fernandez von der Tilburg Universität sowie Paul Schoukens von der KU Leuven fragen in ihrem Beitrag „New Work Forms: How to Integrate them in our Social Insurances?", wie sich Sozialversicherungen in neue Arbeitsformen wie Plattformarbeit integrieren lassen. Aus einer Zunahme der Gruppe atypisch Beschäftigter folgt, dass auch dringend ihre soziale Absicherung gewährleistet werden muss. Die Handlungsnotwendigkeit hängt von dem Ausmaß ab, in dem das atypische Beschäftigungsverhältnis vom etablierten Standard - dem Vollzeitbeschäftigten mit einem unbefristeten Arbeitsvertrag - abweicht. Die Autoren schlagen ein System der sozialen Sicherung vor, das in seiner Umsetzung ausreichend flexibel ist, um den verschiedenen Arbeitnehmergruppen gleichermaßen gerecht zu werden und gleichzeitig ein finanziell tragfähiges System der sozialen Absicherung aufrechtzuerhalten, das typischen und atypischen Beschäftigten einen ausreichenden Sozialversicherungsschutz bieten kann.

Philipp Steinberg und Gero Roser vom Bundesministerium für Wirtschaft und Energie fokussieren ihren Beitrag auf die wirtschaftspolitischen Herausforderungen der Digitalisierung. Diese bringt weitreichende Veränderungen für Unternehmen, Beschäftigte und Verbraucher mit sich. Die damit verbundenen Veränderungen in Wirtschaft und Gesellschaft stellen die Wirtschaftspolitik vor neue Herausforderungen: Sie muss die regulatorischen Rahmenbedingungen anpassen, um Innovationen in neuen Technologien und Anwendungsfeldern zu ermöglichen, die Wettbewerbsfähigkeit Deutschlands auch in einer digitalisierten Welt zu fördern und den Wettbewerb in digitalen Märkten sicherzustellen. Durch Experimentierklauseln, eine bessere digitale Infrastruktur und gezielte Unterstützung von Unternehmen kann sie dafür sorgen, dass Innovationen schneller umgesetzt und auf den Markt gebracht werden können.

In einer abschließenden Podiumsdiskussion diskutierten Julia Borggräfe vom Bundesministerium für Arbeit und Soziales, Michael Hüther, Philipp Steinberg und Enzo Weber die verschiedenen wirtschaftspolitischen Handlungsfelder, auf denen die Politik wirken kann. So wurde die Notwendigkeit staatlicher Unterstützung für Infrastrukturinvestitionen, etwa beim Thema Breitbandausbau, nachdrücklich thematisiert. Auch dürfte Digitalisierung eine erhebliche Rolle für die regionale Entwicklung spielen. Arbeitsmarktpolitisch wurde sowohl die Frage der Kompensationsmöglichkeiten für Verlierer der Digitalisierung als auch die Rolle des Staats bei qualifizierenden Maßnahmen und in der Weiterbildung diskutiert, sodass die Zahl derjenigen, die potenziell zu den „Verlierern“ dieser Entwicklung zählen, minimiert werden. Weiterhin zeigten sich industrie-, wettbewerbs- und steuerpolitisch Regulierungsbedarfe in Hinblick auf multinationale Champions in der ITBranche und der Plattformökonomie, auch weil zahlreiche dieser Champions ihren Sitz im Ausland haben. Einigkeit bestand darüber, dass der Themenkreis „Digitalisierung" als Herausforderung oder Chance auf zahlreichen sehr unterschiedlichen Politikfeldern zu verstehen ist. 